\title{
Práticas de saúde na prevenção das infecções sexualmente transmissíveis
}

\author{
Health practices in the prevention of sexually transmitted infections \\ Prácticas de salud en la prevención de infecciones de transmisión sexual
}

\section{Resumo}

Objetivo: conhecer a produção científica sobre as práticas de saúde na prevenção das infecções sexualmente transmissíveis. Metodologia: estudo descrito, do tipo revisão integrativa. Utilizou-se a Biblioteca Virtual de Saúde para coleta de dados entre dezembro de 2018 a janeiro de 2019. Os dados incluídos foram artigos completos e disponíveis na íntegra, no idioma português, publicados no período de 2013 a 2018. Resultados: foram analisados 12 artigos. As informações foram divididas em duas categorias de acordo com o tipo de estratégia empregada em práticas de prevenção. As escolas se apresentam como o cenário para a realização das ações preventivas e os adolescentes são o principal alvo das intervenções. Constatou-se a dificuldade dos profissionais da área da saúde e educação em abordar temas relacionados à saúde sexual e sexualidade, necessidade de educação permanente aos profissionais. As oficinas e atividades grupais têm impactos positivo no enfrentamento das infecções, porém, o estigma e tabu ainda permeiam o assunto. Conclusão: as ações assistenciais e educativas devem ser realizadas em conjunto. É salutar que haja qualificação e aprimoramento contínuos dos profissionais, organização dos serviços de saúde e incorporação das ações intersetoriais, além de ações na comunidade que promovam a percepção de risco para as infecções sexualmente transmissíveis, com estímulo de práticas seguras para a saúde em todos os grupos e faixas etárias.

Palavras-chave: Infecções sexualmente transmissíveis; Prevenção primária; Atenção básica; Saúde sexual; Enfermagem.

\begin{abstract}
Objective: to know the scientific production on health practices in the prevention of sexually transmitted infections. Methods: described study, of the type integrative review. The Virtual Health Library (VHL) was used for data collection between December 2018 and January 2019. The data included were complete articles and available in full, in Portuguese, published between 2013 and 2018. Results: 12 articles were analyzed, the information was divided into two categories according to the type of strategy employed in prevention practices, namely: assistance actions and preventive educational actions. Schools present themselves as the scenario for carrying out preventive actions and adolescents are the main target of interventions. It was noted the difficulty of health and education professionals in addressing issues related to sexual health and sexuality, the need for continuing education for professionals. The workshops and group activities have a positive impact in the fight against infections, however, the stigma and taboo still permeate the subject. Conclusions: assistance and educational actions must be carried out together. It is salutary that there is continuous qualification and improvement of professionals, organization of health services and incorporation of intersectoral actions, in addition to actions in the community that promote the perception of risk for sexually transmitted infections, with the encouragement of safe practices for health in all countries. groups and age groups.
\end{abstract}


Keywords: Sexually transmitted infections; Primary prevention; Basic care; Sexual health; Nursing.

\section{Resumen}

Objetivo: conocer la producción científica sobre prácticas de salud en la prevención de infecciones de transmisión sexual. Métodos: estudio descrito, del tipo revisión integradora. Para la recolección de datos se utilizó la Biblioteca Virtual en Salud (BVS) entre diciembre de 2018 y enero de 2019. Los datos incluidos fueron artículos completos y disponibles íntegramente, en portugués, publicados entre 2013 y 2018. Resultados: Se analizaron 12 artículos, la información se dividió en dos categorías según el tipo de estrategia empleada en las prácticas de prevención, a saber: acciones asistenciales y acciones educativas preventivas. Las escuelas se presentan como el escenario para la realización de acciones preventivas y los adolescentes son el principal objetivo de las intervenciones. Se señaló la dificultad de los profesionales de la salud y la educación para abordar temas relacionados con la salud sexual y la sexualidad, la necesidad de educación continua para los profesionales. Los talleres y actividades grupales tienen un impacto positivo en la lucha contra las infecciones, sin embargo, el estigma y el tabú aún permean el tema. Conclusiones: las acciones asistenciales y educativas deben realizarse conjuntamente. Es saludable que exista la capacitación y mejora continua de los profesionales, la organización de los servicios de salud y la incorporación de acciones intersectoriales, además de acciones en la comunidad que promuevan la percepción de riesgo de infecciones de transmisión sexual, con el fomento de prácticas seguras para la salud en todos los países, grupos y grupos de edad.

Palabras clave: Infecciones de transmisión sexual; Prevención primaria; Cuidados básicos; Salud sexual; Enfermería.

\section{Introdução}

As Infecções Sexualmente Transmissíveis (IST) têm múltiplas etiologias e são causadas por mais de trinta agentes etiológicos (vírus, bactérias e outros microrganismos) (OMS, 2020). São disseminadas, predominantemente, por contato sexual (vaginal, anal e oral) e podem ser transmitidas através de meios não sexuais como sangue e seus derivados; na transmissão vertical por via transplacentária, no parto e/ou na amamentação (Brasil, 2020a).

As IST podem ser classificadas sob a forma de síndromes de acordo com as apresentações dos sinais e sintomas de úlceras genitais, corrimento uretral, corrimento vaginal, verrugas anogenitais e doença inflamatória pélvica (DIP) (Brasil, 2020a). Contudo, na maioria dos casos, as IST são assintomáticas ou apresentam sintomas benignos que não são reconhecidos como uma infecção, o que pode acarretar sérias consequências imediatas para a saúde reprodutiva como a infertilidade, a transmissão de infecções verticais, e até mesmo óbito (OMS, 2020).

Em todo o mundo estima-se que mais de um milhão de IST são adquiridas diariamente (OMS, 2020). Segundo dados da Organização Mundial da Saúde (OMS) em 2019 havia um total de 38 milhões de pessoas vivendo com o Vírus da Imunodeficiência Humana (HIV), neste ano foram registradas 1,7 milhão de novas infecções por HIV (OMS \& UNAIDS, 2020).

No Brasil, em 2019, foram diagnosticados 41.909 novos casos de HIV (Brasil, 2020b), no que se refere a sífilis nesse mesmo período foram detectados 152.915 casos de sífilis adquirida, 61.127 casos de sífilis em gestantes e 24.130 casos de sífilis congênita (Brasil, 2020c). Com relação as Hepatites virais foram confirmados 13.971 casos de Hepatite B, Hepatite C foram 22.747 casos (Brasil, 2020d). Estima-se que existem cerca de 500 mil novos casos de infecção por gonorreia na população em geral (Brasil, 2016a).

A situação epidemiológica das infecções, em virtude das subnotificações e da ausência de notificação compulsória de algumas IST, não é totalmente conhecida (Brasil, 2014). Vale ressaltar que a gonorreia, clamídia, tricomoníase, Vírus Herpes Simples (HSV) e o Papilomavírus Humano (HPV) não estão incluídos na lista nacional de doenças e agravos de notificação compulsória, esses fatos impedem uma análise epidemiológica mais rigorosa com relação às tendências das infecções no país.

No que se refere às faixas etárias ressaltam a tendência mais acentuada de notificações das IST entre os jovens e adultos no país, principalmente HIV e sífilis adquirida (Brasil, 2020b; Brasil, 2020c). No que concerne aos casos das hepatites virais o maior percentual de casos ocorreu entre pessoas de 30 a 44 anos, havendo aumento nos indivíduos de 60 anos ou mais (Brasil, 2020d). 
Nos últimos anos as IST readquiriram importância como problema de saúde pública e, devido a sua magnitude, transcendência e vulnerabilidade devem ser priorizadas as estratégias de prevenção, pois esses agravos ocasionam impactos sociais, econômicos, familiares e psicológicos, o que envolve culpa, estigma, discriminação e violência (OMS, 2016).

Assim, as ações preventivas são intervenções orientadas a evitar o surgimento de doenças específicas, seu objetivo é o controle da transmissão de doenças infecciosas e a redução do risco de doenças degenerativas ou outros agravos, reduzindo sua incidência e prevalência nas populações. A prevenção em saúde exige uma ação antecipada, baseada no conhecimento da história natural a fim de limitar o progresso posterior da doença (Czeresnia, 2003).

Para o controle e a adequada atenção às IST se adota a prevenção combinada que contempla diversas ações de prevenção e assistência. Uma das estratégias faz uso simultâneo de diferentes abordagens de prevenção (biomédica, comportamental e sócioestrutural) aplicadas em múltiplos níveis (individual, nas parcerias/relacionamentos, comunitário, social) para responder a necessidades específicas de determinados grupos (Brasil, 2020).

Entre os métodos que podem ser combinados, estão: a testagem regular para as IST/HIV; a prevenção da transmissão vertical; o tratamento; a imunização para as hepatites A, B e HPV; programas de redução de danos para usuários de álcool e outras substâncias; profilaxia pré-exposição (PrEP); profilaxia pós-exposição (PEP); e o tratamento de pessoas que convivem com HIV e educação em saúde (OMS, 2020; Brasil, 2020; OMS, 2016).

As estratégias de enfrentamento se deparam com barreiras institucionais e sociais como a falta de conhecimento sobre as IST e suas consequências; a insuficiente educação permanente dos profissionais da saúde; o estigma e o preconceito; o difícil manejo para mudanças das práticas sexuais e na percepção de riscos dos indivíduos; a mudança no perfil da população acometida pelas infecções. Esses aspectos continuam sendo obstáculos para a efetivação das intervenções em saúde tornandoas insuficientes para conter a disseminação e exigindo a intensificação das ações de prevenção na atenção à saúde (OMS, 2020).

Diante disso, formulou-se a questão de pesquisa: Qual a produção científica sobre as práticas de saúde na prevenção das IST no Brasil? E como objetivo: conhecer a produção científica sobre as práticas de saúde na prevenção das infecções sexualmente transmissíveis.

\section{Metodologia}

Trata-se de estudo do tipo revisão integrativa. Este tipo de revisão possibilita sintetizar achados provenientes de estudos primários, onde sua abordagem permite a inclusão de estudos experimentais e não experimentais para uma compreensão completa e rigorosa do fenômeno analisado (Soares et al., 2014).

Levou-se em consideração o rigor metodológico com o processo de elaboração em seis fases: 1) Definição da pergunta norteadora; 2) Busca de dados/amostragem na literatura; 3) Coleta de dados; 4) Organização categórica dos estudos incluídos; 5) Discussão crítico-reflexiva dos resultados; 6) Apresentação da revisão (Mendes et al., 2008).

Para o levantamento dos estudos na literatura estabeleceu-se os seguintes critérios de inclusão: artigos completos e disponíveis na íntegra, no idioma português, que abordassem a temática do estudo sobre as práticas de saúde na prevenção das IST, publicados no período de 2013 a 2018; os critérios de exclusão foram trabalhos do tipo teses, dissertações, cartas ao leitor, editoriais, livros ou capítulos de livros, documentos duplicados, recursos educacionais abertos.

A pesquisa foi realizada utilizando-se busca primária na base de dados do portal da Biblioteca Virtual de Saúde (BVS). O período da coleta de dados ocorreu entre dezembro 2018 a janeiro de 2019. Utilizou-se os seguintes descritores indexados nos Descritores em Ciência da Saúde (DeCS): DST; IST (nova nomenclatura desde 2016); prevenção primária; promoção da saúde. 
Optou-se por utilizar o termo DST como descritor de assunto por sua ampla utilização como termo indexador e pelo fato da nomenclatura IST está em vigor no país apenas a partir de 2016 (Brasil, 2016b). O descritor IST foi descartado. Os descritores foram combinados utilizando-se o operador booleano AND na seguinte ordem: "DST AND Prevenção Primária" (Figura 01) e "DST AND Promoção da Saúde" (Figura 02).

Figura 1 - Fluxograma com resultados da seleção dos artigos conforme cruzamento dos descritores "DST AND Prevenção primária”. Salvador, Bahia, Brasil, 2019.

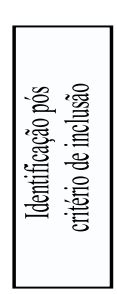

Registros identificados mediante a busca em base de dados $(n=615)$
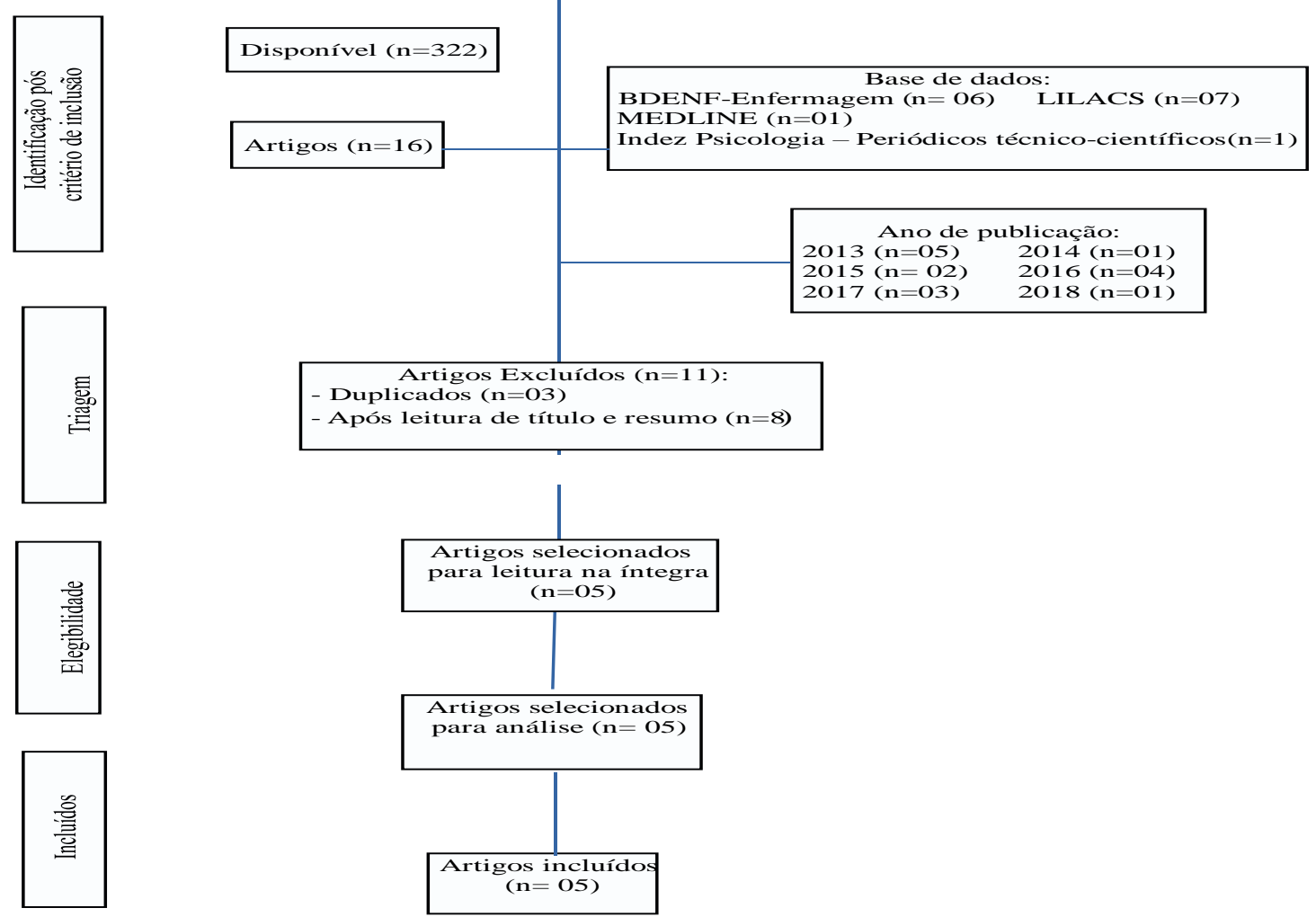

Fonte: Adaptado do modelo Prisma Galvão, Pansani \& Harrad, (2015). 
Figura 2 - Fluxograma com resultados da seleção dos artigos conforme cruzamento dos descritores "DST AND Promoção da Saúde”. Salvador, Bahia, Brasil, 2019.

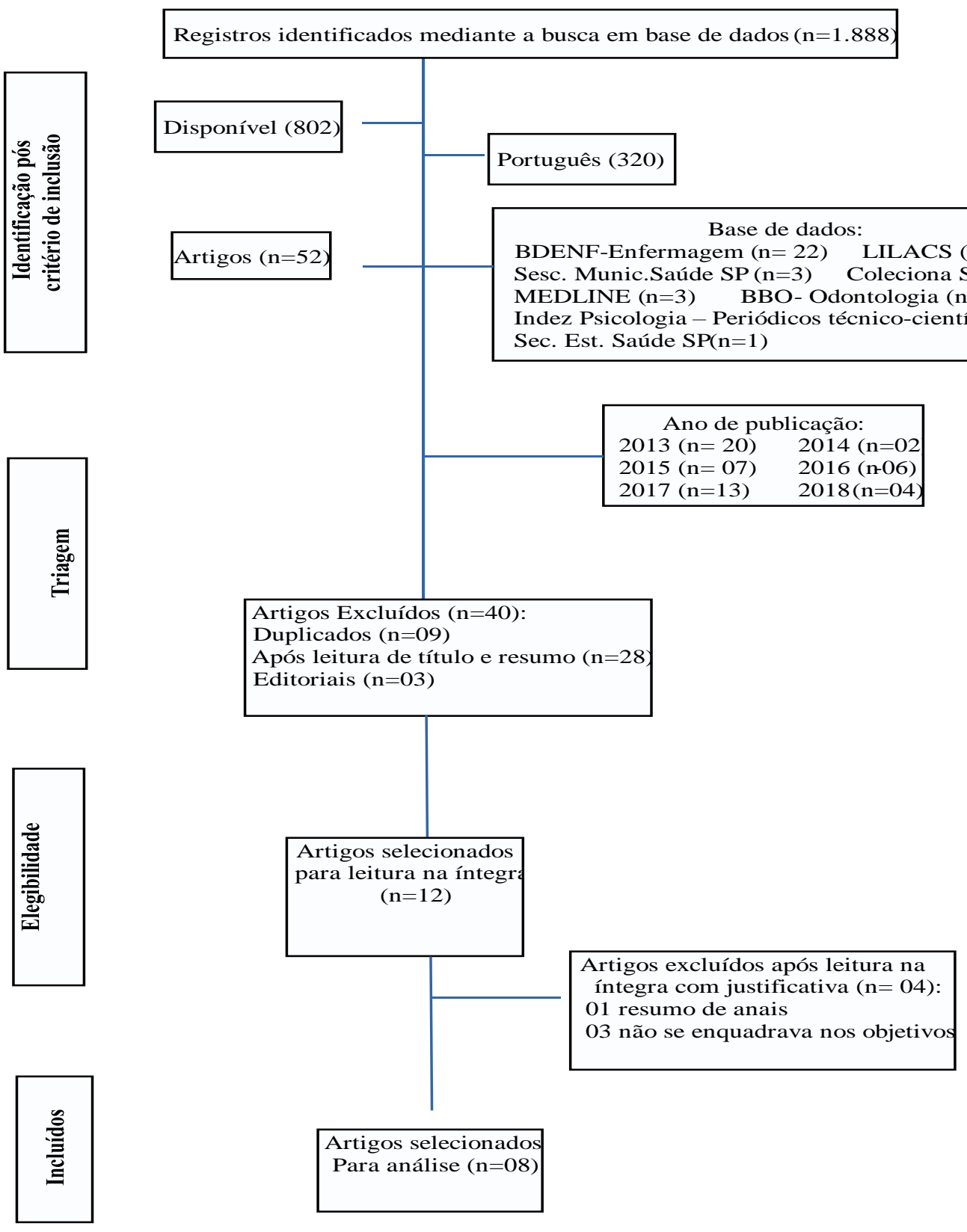

Fonte: Adaptado do modelo Prisma Galvão, Pansani \& Harrad, (2015).

Os resultados da etapa de busca foram dispostos em um quadro contendo variáveis relacionadas à identificação dos artigos como título, autores, ano e periódico de publicação, cenário da pesquisa, local do estudo, metodologia, objetivo e população da pesquisa (faixa etária) e síntese dos principais resultados.

Para análise das informações coletadas, utilizou-se a técnica de agrupamento e categorização. Em virtude da natureza desse estudo não houve necessidade de aprovação no Comitê de Ética em Pesquisa (CEP). 


\section{Resultados}

Nesta revisão integrativa foram analisados 12 artigos. Quanto ao tipo de delineamento dos estudos avaliados, evidenciou-se na amostra o predomínio de artigos qualitativos (n=08) e estudo do tipo relato de experiência (n=04). Entres eles, oito (08) foram publicados em revistas com área de concentração em enfermagem (Rodrigues et al., 2016; Xavier et al., 2017; Cordeiro et al., 2017; Carvalho et al., 2013; Santos et al., 2017; Angelim, 2016; Reis et al., 2014 \& Silva, 2013) totalizando 66,6\% das publicações encontradas, seguidos de uma na área interdisciplinar (Torres et al., 2015), um de saúde coletiva (Russo \& Arreguy, 2015), um de saúde pública (Nasser et al., 2017) e um em psicologia (Ferraz \& Nemes, 2013).

Com relação ao ano de publicação houve concentração nos anos de 2013 e 2017 com 50\% das publicações, quatro artigos em cada ano. No ano de 2016 houve duas publicações, uma em 2014 e uma em 2015. Observa-se que a região Nordeste do Brasil foi o lócus de estudo de nove artigos.

As escolas foram o principal cenário para a realização das ações preventivas sobre a temática e os adolescentes o público-alvo das intervenções (Torres et al., 2015; Cordeiro et al., 2017; Santos et al., 2017; Angelim et al., 2016; Silva et al., 2013; Russo \& Arreguy, 2015). As Unidades Básicas de Saúde (UBS) apareceram em segundo lugar, com ações de prevenção direcionadas para as mulheres apontando questões de gênero que permeiam o cuidar da saúde no enfrentamento às IST (Rodrigues et al., 2015; Ferraz \& Nemes, 2013; OMS e UNAIDS, 2020; Xavier et al., 2017 \& Carvalho, 2013). Um estudo apresentou ações preventivas direcionadas a mulheres profissionais do sexo que foram realizadas em locais como bares, postos de gasolina e pontos de encontros pré-estabelecido entre as educadoras sociais e as mulheres (Reis, 2014).

A síntese dos artigos e suas variáveis estão apresentadas no quadro 01.

Quadro 1 - Síntese dos artigos sobre as práticas de saúde na prevenção das infecções sexualmente transmissíveis no Brasil.

Salvador, Bahia, Brasil, 2019.

\begin{tabular}{|c|c|c|c|c|c|c|c|}
\hline Título & Autores & $\begin{array}{c}\text { Ano de } \\
\text { publicação }\end{array}$ & $\begin{array}{l}\text { Base de } \\
\text { dados }\end{array}$ & $\begin{array}{c}\text { Cenário da } \\
\text { pesquisa/Local }\end{array}$ & $\begin{array}{c}\text { Tipo de } \\
\text { estudo/Objetivo }\end{array}$ & $\begin{array}{l}\text { População } \\
\text { (N) }\end{array}$ & Principais achados \\
\hline $\begin{array}{l}\text { Atuação de } \\
\text { enfermeiros no } \\
\text { acompanhamento } \\
\text { da sífilis na } \\
\text { atenção primária }\end{array}$ & $\begin{array}{l}\text { Rodrigues, } \\
\text { A.R.M et } \\
\text { al. }\end{array}$ & 2016 & BDENF & $\begin{array}{l}\text { Centro de saúde. } \\
\text { Sobral-CE }\end{array}$ & $\begin{array}{l}\text { Pesquisa } \\
\text { qualitativa. } \\
\text { Analisar a atuação } \\
\text { de enfermeiros na } \\
\text { APS no } \\
\text { acompanhamento } \\
\text { da sífilis. }\end{array}$ & $\begin{array}{l}\text { Enfermeiros } \\
(\mathrm{n}=18)\end{array}$ & $\begin{array}{l}\text { Dificuldade dos } \\
\text { enfermeiros em } \\
\text { discutir a vivência da } \\
\text { sexualidade devido às } \\
\text { questões de gênero } \\
\text { envolvidas e ao } \\
\text { estigma. }\end{array}$ \\
\hline $\begin{array}{l}\text { Avaliação na } \\
\text { atenção primária } \\
\text { paulista: ações } \\
\text { Incipientes em } \\
\text { saúde sexual e } \\
\text { reprodutiva }\end{array}$ & $\begin{array}{l}\text { Nasser, } \\
\text { M.A et al. }\end{array}$ & 2017 & MEDLINE & $\begin{array}{l}\text { Atenção Primária } \\
\text { em Saúde - APS } \\
586 \text { municípios } \\
\text { de São Paulo }\end{array}$ & $\begin{array}{l}\text { Pesquisa } \\
\text { avaliativa. } \\
\text { Avaliar o } \\
\text { desempenho dos } \\
\text { serviços de APS do } \\
\text { SUS em ações de } \\
\text { Saúde Sexual e } \\
\text { Reprodutiva } \\
\text { (SSR). }\end{array}$ & $\begin{array}{l}\text { Serviços de } \\
\text { APS }(n= \\
2.735)\end{array}$ & $\begin{array}{l}\text { A implementação da } \\
\text { saúde sexual e } \\
\text { reprodutiva na } \\
\text { atenção primária à } \\
\text { saúde nos serviços } \\
\text { estudados é incipiente. } \\
\text { Deve-se investir em } \\
\text { tecnologias leves e } \\
\text { educação permanente. }\end{array}$ \\
\hline $\begin{array}{l}\text { Câncer de colo } \\
\text { uterino e infecção } \\
\text { sexualmente } \\
\text { transmissível: } \\
\text { percepção das } \\
\text { mulheres privadas } \\
\text { de liberdade }\end{array}$ & $\begin{array}{l}\text { Xavier, L. } \\
\text { D. A. et al. }\end{array}$ & 2017 & BDENF & $\begin{array}{l}\text { Colônia penal } \\
\text { feminina } \\
\text { Recife } / \mathrm{Pe}\end{array}$ & $\begin{array}{l}\text { Pesquisa } \\
\text { Descritiva- } \\
\text { qualitativa. } \\
\text { Investigar a } \\
\text { percepção de } \\
\text { mulheres privadas } \\
\text { de liberdade } \\
\text { quanto à prevenção } \\
\text { do câncer do colo } \\
\text { do útero e IST. }\end{array}$ & $\begin{array}{l}\text { Detentas } \\
(\mathrm{n}=25)\end{array}$ & $\begin{array}{l}\text { Necessidade de } \\
\text { melhorias na } \\
\text { assistência no âmbito } \\
\text { carcerário, bem como } \\
\text { na distribuição de } \\
\text { métodos de proteção e } \\
\text { divulgação de } \\
\text { informações } \\
\text { possibilitando } \\
\text { condições de adotar } \\
\text { medidas preventiva. }\end{array}$ \\
\hline
\end{tabular}




\begin{tabular}{|c|c|c|c|c|c|c|c|}
\hline $\begin{array}{l}\text { Cogestão e } \\
\text { prevenção das } \\
\text { dst/aids na atenção } \\
\text { primária: } \\
\text { Contribuições } \\
\text { desde o referencial } \\
\text { dos direitos } \\
\text { humanos } \\
\text { e da } \\
\text { vulnerabilidade }\end{array}$ & $\begin{array}{l}\text { Ferraz, D A } \\
\text { S e Nemes, } \\
\text { M.I.B. }\end{array}$ & 2013 & $\begin{array}{l}\text { Psicologia - } \\
\text { Periódicos } \\
\text { técnico - } \\
\text { científico. }\end{array}$ & $\begin{array}{l}\text { UBS } \\
\text { São Paulo/SP }\end{array}$ & $\begin{array}{l}\text { Estudo descritivo } \\
\text { Analisar as } \\
\text { potencialidades } \\
\text { dos espaços de } \\
\text { cogestão sobre } \\
\text { prevenção das } \\
\text { DST/aids nos } \\
\text { serviços de atenção } \\
\text { primária à saúde. }\end{array}$ & $\begin{array}{l}\text { 03 ACS }(\mathrm{n}= \\
03) \\
\text { Enfermeiro } \\
(01) \\
\text { Médico (01) } \\
\text { Aux.Enf. (02) } \\
\text { Coord. do } \\
\text { Progr. De } \\
\text { DST (01) }\end{array}$ & $\begin{array}{l}\text { Reconhece a } \\
\text { potencialidade } \\
\text { tecnológica do } \\
\text { aconselhamento pré- } \\
\text { teste, aproveitando a } \\
\text { oportunidade da } \\
\text { demanda, e a } \\
\text { necessidade de } \\
\text { formação e } \\
\text { capacitação dos } \\
\text { profissionais, } \\
\text { reorganização dos } \\
\text { serviços e processo de } \\
\text { trabalhos. }\end{array}$ \\
\hline $\begin{array}{l}\text { Comunicação em } \\
\text { saúde: uso de uma } \\
\text { web rádio com } \\
\text { escolares }\end{array}$ & $\begin{array}{l}\text { Torres, } \\
\text { R.A.M. }\end{array}$ & 2013 & LILACS & $\begin{array}{l}\text { Escola de ensino } \\
\text { médio } \\
\text { Sobral Ceará }\end{array}$ & $\begin{array}{l}\text { Relato de } \\
\text { experiência. } \\
\text { Descrever a } \\
\text { vivência dos } \\
\text { graduandos de } \\
\text { enfermagem na } \\
\text { promoção do } \\
\text { cuidado em saúde } \\
\text { com os jovens } \\
\text { escolares acerca } \\
\text { das DST/AIDS. }\end{array}$ & $\begin{array}{l}\text { Estudantes } \\
(\mathrm{n}=12)\end{array}$ & $\begin{array}{l}\text { Observou-se o } \\
\text { interesse e a interação } \\
\text { entre os estudantes } \\
\text { com os temas } \\
\text { abordados, assim } \\
\text { como, a importância } \\
\text { da discussão com os } \\
\text { jovens por meio da } \\
\text { web rádio, quebrando } \\
\text { barreiras, favorecendo } \\
\text { a comunicação acerca } \\
\text { das doenças e um } \\
\text { diálogo horizontal e } \\
\text { dinâmico, facilitando } \\
\text { à expressão da fala. }\end{array}$ \\
\hline $\begin{array}{l}\text { Adolescentes } \\
\text { escolares acerca } \\
\text { das dst/aids: } \\
\text { quando o } \\
\text { conhecimento não } \\
\text { acompanha as } \\
\text { práticas seguras }\end{array}$ & $\begin{array}{l}\text { Cordeiro } \\
\text { J.K.R, et al. }\end{array}$ & 2017 & $\begin{array}{l}\text { Rev Enferm } \\
\text { UFPE }\end{array}$ & $\begin{array}{l}\text { Escolas de } \\
\text { ensino médio } \\
\text { Parelhas/RN }\end{array}$ & $\begin{array}{l}\text { Estudo transversal, } \\
\text { de abordagem } \\
\text { quantitativa; } \\
\text { Avaliar os saberes } \\
\text { e as práticas de } \\
\text { adolescentes em } \\
\text { relação às } \\
\text { DST/AIDS. }\end{array}$ & $\begin{array}{l}\text { Estudantes } \\
(\mathrm{n}=140) .\end{array}$ & $\begin{array}{l}\text { O estudo ressaltou } \\
\text { discreta participação } \\
\text { do PSE nas ações de } \\
\text { saúde. Necessidade de } \\
\text { ações articuladas entre } \\
\text { as instituições, além } \\
\text { de uma abordagem } \\
\text { mais ampla sobre a } \\
\text { sexualidade, práticas } \\
\text { sexuais seguras e } \\
\text { atenção integral ao } \\
\text { indivíduo. }\end{array}$ \\
\hline $\begin{array}{l}\text { Prevenção de } \\
\text { doenças } \\
\text { sexualmente } \\
\text { transmissíveis por } \\
\text { mulheres } \\
\text { Homossexuais e } \\
\text { bissexuais: estudo } \\
\text { descritivo }\end{array}$ & $\begin{array}{l}\text { Carvalho P. } \\
\text { M. G et al. }\end{array}$ & 2013 & LILACS & $\begin{array}{l}\text { Grupo de apoio } \\
\text { às LGBT. } \\
\text { Teresina, Piauí. }\end{array}$ & $\begin{array}{l}\text { Pesquisa } \\
\text { exploratório, } \\
\text { descritivo e } \\
\text { qualitativo. } \\
\text { Identificar a } \\
\text { percepção das } \\
\text { mulheres } \\
\text { homossexuais e } \\
\text { bissexuais sobre a } \\
\text { assistência e } \\
\text { formas de prevenir } \\
\text { as DST. }\end{array}$ & $\begin{array}{l}\text { Mulheres } \\
\text { Lésbicas e } \\
\text { bissexuais do } \\
\text { grupo de } \\
\text { apoio às } \\
\text { LGBT (n= } \\
09)\end{array}$ & $\begin{array}{l}\text { Propõe-se um diálogo } \\
\text { entre os serviços de } \\
\text { saúde e está } \\
\text { população para } \\
\text { identificar } \\
\text { necessidades de saúde } \\
\text { e promover ações para } \\
\text { prevenção de doenças } \\
\text { e promoção da saúde. }\end{array}$ \\
\hline $\begin{array}{l}\text { Pré-carnaval } \\
\text { educativo sobre } \\
\text { infecções } \\
\text { sexualmente } \\
\text { transmissíveis com } \\
\text { adolescentes } \\
\text { escolares }\end{array}$ & $\begin{array}{l}\text { Santos. } \\
\text { M.P et al. }\end{array}$ & 2017 & $\begin{array}{l}\text { Rev Enferm } \\
\text { UFPE }\end{array}$ & $\begin{array}{l}\text { Escola de nível } \\
\text { Médio/Técnico } \\
\text { Maciço do } \\
\text { Baturité/CE }\end{array}$ & $\begin{array}{l}\text { Pesquisa } \\
\text { Qualitativa. } \\
\text { Relatar a } \\
\text { experiência da } \\
\text { condução de uma } \\
\text { oficina com } \\
\text { adolescentes } \\
\text { acerca das IST. }\end{array}$ & $\begin{array}{l}\text { Estudantes } \\
(\mathrm{n}=34)\end{array}$ & $\begin{array}{l}\text { O estudo ressalta a } \\
\text { importância da } \\
\text { educação em saúde de } \\
\text { forma contínua e que } \\
\text { as oficinas tipo rodas } \\
\text { de conversas geram } \\
\text { autorreflexão visando } \\
\text { desenvolver a } \\
\text { autonomia e o } \\
\text { protagonismo dos } \\
\text { adolescentes a }\end{array}$ \\
\hline
\end{tabular}




\begin{tabular}{|c|c|c|c|c|c|c|c|}
\hline & & & & & & & $\begin{array}{l}\text { respeito da saúde } \\
\text { sexual. }\end{array}$ \\
\hline $\begin{array}{l}\text { Atividades } \\
\text { educativas sobre } \\
\text { práticas sexuais de } \\
\text { risco para } \\
\text { estudantes: relato } \\
\text { de experiência }\end{array}$ & $\begin{array}{l}\text { Angelim, } \\
\text { R.C.M et } \\
\text { al. }\end{array}$ & 2016 & $\begin{array}{l}\text { Rev Enferm } \\
\text { UFPI. }\end{array}$ & $\begin{array}{l}\begin{array}{l}\text { Escola de ensino } \\
\text { médio }\end{array} \\
\text { Rescife/Pe }\end{array}$ & $\begin{array}{l}\text { Estudo descritivo } \\
\text { do tipo relato de } \\
\text { experiência. } \\
\text { Relatar a } \\
\text { experiência de } \\
\text { graduandos de } \\
\text { enfermagem } \\
\text { quanto as } \\
\text { atividades } \\
\text { educativas sobre } \\
\text { sexualidade e } \\
\text { práticas sexuais } \\
\text { direcionadas a } \\
\text { estudantes de nível } \\
\text { médio. }\end{array}$ & $\begin{array}{l}\text { Estudantes } \\
(\mathrm{n}=200)\end{array}$ & $\begin{array}{l}\text { Observa-se a } \\
\text { necessidade de } \\
\text { incorporar atividades } \\
\text { educativas acerca das } \\
\text { DST com o intuito de } \\
\text { incentivar os } \\
\text { estudantes a refletir e } \\
\text { compreender a } \\
\text { necessidade de } \\
\text { realizar práticas } \\
\text { sexuais saudáveis. }\end{array}$ \\
\hline $\begin{array}{l}\text { Educação em } \\
\text { saúde com } \\
\text { prostitutas: uma } \\
\text { experiência de } \\
\text { educação aos pares }\end{array}$ & $\begin{array}{l}\text { Reis, T.G.O } \\
\text { et al. }\end{array}$ & 2014 & $\begin{array}{l}\text { Rev Enferm } \\
\text { UFPI. }\end{array}$ & $\begin{array}{l}\text { Associação das } \\
\text { Profissionais do } \\
\text { Sexo } \\
\text { Picos-PI - PI }\end{array}$ & $\begin{array}{l}\text { Estudo de caso } \\
\text { descritivo; } \\
\text { Conhecer a } \\
\text { vivência de } \\
\text { educadoras sociais } \\
\text { sobre atividades de } \\
\text { educação em saúde } \\
\text { com prostitutas. }\end{array}$ & $\begin{array}{l}\text { Ex- } \\
\text { profissionais } \\
\text { do sexo e } \\
\text { educadoras } \\
\text { sociais }\end{array}$ & $\begin{array}{l}\text { Conclui-se a } \\
\text { necessidade de } \\
\text { programas de } \\
\text { intervenção mais } \\
\text { eficazes que facilitem } \\
\text { o trabalho educativo } \\
\text { bem como } \\
\text { capacitações para a } \\
\text { realização de } \\
\text { educação aos pares. }\end{array}$ \\
\hline $\begin{array}{l}\text { Sexualidade na } \\
\text { adolescência: } \\
\text { relato de } \\
\text { experiência }\end{array}$ & $\begin{array}{l}\text { Silva, D.M } \\
\text { et al. }\end{array}$ & 2013 & $\begin{array}{l}\text { Rev enferm } \\
\text { UFPE on } \\
\text { line }\end{array}$ & $\begin{array}{l}\begin{array}{l}\text { Escola do ensino } \\
\text { médio }\end{array} \\
\text { Jequié/BA }\end{array}$ & $\begin{array}{l}\text { Estudo descritivo, } \\
\text { tipo Relato de } \\
\text { experiência. } \\
\text { Descrever a } \\
\text { experiência em } \\
\text { relação a uma } \\
\text { oficina sobre } \\
\text { métodos } \\
\text { contraceptivos e } \\
\text { prevenção de DST. }\end{array}$ & $\begin{array}{l}\text { Estudantes } \\
(\mathrm{n}=31)\end{array}$ & $\begin{array}{l}\text { Concluiu a } \\
\text { necessidade de } \\
\text { implementação de } \\
\text { estratégias educativas } \\
\text { que utilizem } \\
\text { metodologias } \\
\text { participativas, tais } \\
\text { como oficinas, para } \\
\text { que haja um incentivo } \\
\text { a participação e a } \\
\text { conscientização dos } \\
\text { adolescentes sobre a } \\
\text { importância da } \\
\text { prevenção das DST e } \\
\text { da gravidez na } \\
\text { adolescência. }\end{array}$ \\
\hline $\begin{array}{l}\text { Projeto "Saúde e } \\
\text { Prevenção nas } \\
\text { Escolas": } 501 \\
\text { percepções de } \\
\text { professores e } \\
\text { alunos sobre a } \\
\text { distribuição de } \\
\text { preservativos } \\
\text { masculinos no } \\
\text { ambiente escolar }\end{array}$ & $\begin{array}{l}\text { Russo K. e } \\
\text { Arreguy, } \\
\text { M.E. }\end{array}$ & 2015 & LILACS & $\begin{array}{l}\text { Escola Pública } \\
\text { do ensino médio } \\
\text { Rio de } \\
\text { Janeiro/RJ }\end{array}$ & $\begin{array}{l}\text { Estudo descritivo } \\
\text { qualitativo; } \\
\text {-Discutir a } \\
\text { proposta de } \\
\text { distribuição de } \\
\text { preservativos } \\
\text { masculinos nas } \\
\text { escolas. }\end{array}$ & $\begin{array}{l}\begin{array}{l}\text { Estudantes } \\
(\mathrm{n}=18)\end{array} \\
\text { Professores } \\
(\mathrm{n}=12)\end{array}$ & $\begin{array}{l}\text { Os resultados sugerem } \\
\text { que professores estão } \\
\text { distantes dos alunos e } \\
\text { da política pública no } \\
\text { que se refere a } \\
\text { abordagem do tema da } \\
\text { sexualidade e } \\
\text { distribuição do } \\
\text { preservativo } \\
\text { masculino nas escolas. }\end{array}$ \\
\hline
\end{tabular}

Fonte: Autores.

\section{Discussão}

Diante do objetivo desta revisão e das obras utilizadas discutiu-se sobre intervenções de prevenção, estas que se configuram como métodos e meios para auxiliar o sujeito ou grupos sociais à obtenção de informações e conhecimentos sobre IST, de maneira a torná-los capazes de integrar e realizar as várias estratégias de prevenção disponíveis (Brasil, 2017). Com 
bases na ocorrência dos principais relatos, identificou-se duas categorias teóricas: Práticas de saúde em IST: uso de tecnologias como estratégias de prevenção; Atenção integral às IST: um trabalho contínuo e sistemático.

\section{Práticas de saúde em IST: uso de tecnologias como estratégias de prevenção}

As estratégias de prevenção devem ser aprimoradas, levando-se em consideração o acúmulo teórico recente, as novas tecnologias de prevenção surgidas nos últimos anos e as especificidades de cada grupo social (Grangeiro et al., 2015). As práticas de prevenção encontradas nos estudos foram divididas em dois grupos, de acordo o tipo de estratégia empregada: ações assistenciais e ações educativas preventivas.

As ações assistenciais englobam as medidas de proteção específicas e individuais como a vacinação contra Hepatite B e HPV, a coleta para o citopatológico oncótico, a oferta e distribuição de preservativos, a realização de testagem sorológicas e aconselhamento pré e pós testes, o tratamento, acompanhamento e notificação dos casos. Esse tipo de estratégia foi predominante no estudo que avaliou serviços de Atenção Primária em Saúde (APS) e percepção dos profissionais no cuidado (Rodrigues et al, 2016; Nasser et al., 2017; Xavier et al., 2017; Ferraz \& Nemes, 2013; Carvalho et al., 2013).

Com relação a percepção dos usuários os estudos demonstram que a prevenção é ofertada por meio de exames ginecológico e do rastreamento do câncer de colo do útero, cuidados com a higiene (Xavier et al., 2017; Carvalho et al., 2013). Não foi citado por nenhum dos estudos o uso/distribuição de preservativos como método de barreira para as infecções ao se constituir como meio para prevenção de doenças e terapia combinatória em tratamentos específicos das IST.

A oferta e incentivo ao uso de preservativo é considerada uma intervenção padrão, considerado efetivo, seguros, de baixo custo e sem efeitos colaterais que configuram também uma estratégia de contracepção.

No que concerne as ações educativas preventivas, essas compreendiam a promoção da saúde e possuíam um caráter educativo abrangente dirigidas a grupos específicos (Torres et al., 2015; Santos et al., 2017; Angelim et al.,2016; Reis et al., 2014; Silva et al., 2013). As ações educativas como orientações em sala de espera, palestras com distribuição de preservativos e materiais educativos, assim como as atividades de grupos foram as principais estratégias de intervenções utilizadas por profissionais da saúde (Rodrigues et al., 2016; Reis et al, 2014). Os professores utilizam palestras e/ou aulas regulares como meio de trabalhar temas sobre práticas sexuais e IST, porém, restritos a aspectos biológicos que envolvem a temática (Russo \& Arreguy, 2015).

Percebe-se nos estudos selecionados que as oficinas em saúde foram utilizadas como o método habitual nas ações intersetoriais direcionados aos jovens escolares, com temáticas voltadas ao esclarecimento sobre à puberdade, a sexualidade, reflexão das relações de gênero e, principalmente às ações preventivas relacionadas às práticas sexuais de risco para as IST (Torres et al., 2015; Santos et al., 2017; Angelim et al., 2016; Silva et al., 2013; Costa \& Silva et al., 2016). Como recursos para o desenvolvimento das ações educativas predominou o vídeo educativo, dinâmica de perguntas e respostas com balões, caixa surpresa e aula expositiva abordando as principais IST (Angelim et al.,2016; Costa \& Silva et al., 2016).

As ações assistenciais foram mais utilizadas quando comparadas as ações de educação em saúde e ocorreram de maneira pontual (Rodrigues et al, 2016; Nasser et al., 2017; Cordeiro et al., 2017; Reis et al., 2014). Apesar de reconhecerem a potencialidade das ações educativas os profissionais priorizaram ações individuais nas unidades de saúde e nos consultórios, o que reafirma a ênfase no modelo biomédico centrado.

Vale ressaltar, a potencialidade em realização de oficinas e rodas de conversas como meios profícuos para gerar autorreflexão, autonomia e o protagonismo de sujeitos frente à saúde sexual (Santos et al., 2017). As intervenções cujo foco está no comportamento buscam favorecer a adoção de conhecimentos e informações para evitar exposição às IST, bem como, desenvolver estratégias de enfrentamento para melhorar a capacidade de gerir os diferentes graus de riscos a que estão expostos (Brasil, 2017). As práticas preventivas com ênfase em IST devem articular ações individuais e coletivas, ações 
intersetoriais, recursos materiais e humanos, tecnologias e estratégias para o enfrentamento das necessidades de saúde do sujeito e comunidade.

\section{Atenção integral às IST: um trabalho contínuo e sistemático}

A atenção básica desenvolve ações de prevenção dos agravos e de promoção à saúde de maneira contínua e resolutiva. Entretanto, a atenção integral ao enfretamento de afecções necessita não apenas da implementação de ações elementares de prevenção e assistência, mas da articulação, fortalecimento e integração entre os diferentes níveis de atenção à saúde (OMS, 2016).

Considera-se que as ações preventivas são importantes para o enfrentamento de agravos. Alguns estudos identificaram que os profissionais da saúde se sentem despreparados para atuar frente aos aspectos sociais, psicológicos, culturais, religiosos e sexuais que abrangem o sujeito, ressaltando a necessidade de intensificar o processo de qualificação.

É necessário rever finalidades do serviço em saúde e organizar a gestão do trabalho, disseminar tecnologias e investir em educação permanente (Nasser et al., 2017; Xavier et al., 2017; Ferraz \& Nemes, 2013). Pesquisa revela que alguns profissionais têm dificuldade em superar o modelo de prevenção normativo individual, apesar de considerar a prevenção o componente central do seu trabalho e as orientações preventivas como parte importante dos atendimentos (Ferraz \& Nemes, 2013). Na percepção de usuários, os profissionais de saúde têm dificuldade em escutar e acolher suas demandas (OMS, 2020).

Corroborando com esses achados, os demais estudos ressaltaram a necessidade de repensar e reformular estratégias e atividades de ações preventivas, bem como o processo de formação e educação permanente dos profissionais da saúde e professores (Rodrigues et al., 2016; Carvalho et al., 2013; Russo \& Arreguy 2015). As ações de prevenção das IST poderiam ser mais efetivas se os profissionais levassem em conta a singularidade e o contexto social de cada usuário(a), assim como, o uso adequado/racional das tecnologias disponíveis (Ferraz \& Nemes, 2013).

\section{Conclusão}

Observou-se que a interação com a população alvo de cada estudo ocorreu quando os assuntos propostos eram expostos de maneira dinâmica, como em oficinas, atividades grupais e interativas. Essas ações promovem a troca de saberes e experiências em um ambiente acolhedor, que estimula um diálogo livre com integração, participação e autonomia do sujeito.

As temáticas relacionadas ao diagnóstico precoce, o aconselhamento pré e pós testagem e a terapia adequada para redução do risco de infecções e seus agravos foram abordadas por profissionais da saúde e educação no intuito de qualificar e prestar uma assistência livre de preconceitos sobre sexualidade, comportamento sexual e gênero.

Os estudos apontaram ser possível planejar ações educativas eficientes, capazes de sensibilizar para evitar comportamentos de riscos, considerando o sujeito e o contexto biopsicossocial na construção de estratégias de autocuidado, práticas seguras e corresponsabilidade. Assim, espera-se que haja maior produção científica acerca da prevenção das IST, sobretudo em outros contextos sociais e políticos.

\section{Referências}

Angelim, R. C. M., et al (2016). Atividades educativas sobre práticas sexuais de risco para estudantes: relato de experiência. Rev Enferm UFPI. 5(1),96-100.

Brasil. (2020a). Ministério da Saúde. Secretaria de Vigilância em Saúde. Protocolo Clínico e Diretrizes Terapêuticas para Atenção Integral as Pessoas com Infecções Sexualmente Transmissíveis. Brasília: Ministério da Saúde.

Brasil.(2020b), Ministério da Saúde. Secretaria de Vigilância em Saúde. Departamento de DST/ AIDS e Hepatites Virais. Boletim Epidemiológico HIV/AIDS. Brasília: Ministério da Saúde dez,num. especial 2020. 
Brasil. (2020c), Ministério da Saúde. Secretaria de Vigilância em Saúde. Departamento de DST/AIDS e Hepatites Virais. Boletim Epidemiológico de Sífilis. Brasília: Ministério da Saúde,num. especial 2020.

Brasil. (2020d). Ministério da Saúde. Secretaria de Vigilância em Saúde. Departamento de DST/AIDS e Hepatites Virais. Boletim Epidemiológico das Hepatites virais 2018. Brasília: MS. dez., num. especial 2020.

Brasil. (2017) Ministério da Saúde. Secretaria de Vigilância em Saúde. Departamento de Vigilância, Prevenção e Controle das IST, do HIV/Aids e das Hepatites Virais. Prevenção Combinada do HIV/ Bases conceituais para profissionais, trabalhadores(as) e gestores(as) de saúde. MS - Brasília: Ministério da Saúde.

Brasil. (2016a). Ministério da Saúde. Departamento de Vigilância, Prevenção e Controle das IST, do HIV/Aids e das Hepatites Virais. Pesquisa brasileira sobre resistência do gonococo a medicamentos corrobora dados da OMS e sugere alternativas para o tratamento. Brasília: MS.

Brasil. (2016b). Mistério da Saúde. Departamento de Vigilância, Prevenção e Controle das IST, do HIV/Aids e das Hepatites Virais. Decreto nº 8.901/2016 publicada no Diário Oficial da União em 11.11.2016, Seção I, páginas 03 a 17. Mudança da nomenclatura "IST" (infecções sexualmente transmissíveis) no lugar de "DST" (doenças sexualmente transmissíveis).

Brasil. (2014). Ministério da Saúde. Gabinete do Ministro. PORTARIA N ${ }^{\circ}$ 1.984, DE 12 de setembro de 2014. Lista nacional de doenças e agravos de notificação compulsória. Brasília: MS

Carvalho, P. M. G., et al. (2013). Prevenção de doenças sexualmente transmissíveis por mulheres homossexuais e bissexuais: estudo descritivo. Rev brasileira de enfermagem [Online],12 (4).

Costa e Silva, S. P., Barbosa, A. P. P., Araújo, C. S., et al. (2016) Discutindo Sexualidade/IST no contexto escolar: Práticas de professores de escolas públicas. Rev Enferm UFPE. 10(5):4295-303

Cordeiro, J. K. R., Santos, M. M., Sales, L. K. O., et al. (2017). Adolescentes escolares acerca das DST/Aids: quando o conhecimento não acompanha as práticas seguras. Rev enferm UFPE. 11(7), 2888-96.

Czeresnia, D. (2003). O conceito de saúde e a diferença entre prevenção e promoção [versão revisada e atualizado]. Promoção da saúde: conceitos, reflexões, tendências. Cad. Saúde Pública. 39 (53). Fiocruz

Ferraz, D. A. S., \& Nemes, M. I. B. (2013). Cogestão e Prevenção das DST/Aids na Atenção Primária: contribuições desde o referencial dos direitos humanos e da Vulnerabilidade. Temas em Psicologia. 21 (3). 735-749.

Galvão, T. F., Pansani, T. S, A., \& Harrad, D. (2015). Principais itens para relatar Revisões sistemáticas e Meta-análises: A recomendação PRISMA. Epidemiol. Serv. Saúde. 24(2), 335-342.

Grangeiro, A. et al. (2015). O efeito dos métodos preventivos na redução do risco de infecção pelo HIV nas relações sexuais e seu potencial impacto em âmbito populacional: uma revisão da literatura. Rev Bras Epidemiol 18(01): 43-62.

Mendes, K. D. S, Silveira, R. C. C. P., \& Galvão, C. M. (2008). Revisão integrativa: método de pesquisa para a incorporação de evidências na saúde e na enfermagem. Texto \& Contexto-Enfermagem. 17(4): 758-764

Nasser, M. A et al. (2017) Avaliação na atenção primária paulista: ações incipientes em saúde sexual e reprodutiva. Rev Saúde Pública. 51 (1).

OMS \& UNAIDS. (2020). Organização Mundial de Saúde. Relatório informativo- dez 2020. UN oficial report Fact Sheet.

Organização Mundial da Saúde. Estratégia do setor de saúde global sobre infecções sexualmente transmissíveis, 2016-2021. Organização Mundial da Saúde.

Organização Mundial de Saúde. (2020). Infecções Sexualmente Transmissíveis (DSTs). UN oficial report Fact Sheet. Organização Mundial da Saúde.

Reis, T. G. O et al. (2014). Educação em saúde com prostitutas: uma experiência de educação aos pares. Rev Enferm UFPI. 3(3):46-52

Rodrigues, A. R. M, Silva, M. A. M, Cavalcante, A. E. S, et al. (2016). Atuação de enfermeiros no acompanhamento da sífilis na atenção primária. Rev enferm UFPE. 10(4), 1247-55.

Russo, K., \& Arreguy, M. E. (2015). Projeto "Saúde e Prevenção das Escolas": percepções de professores e alunos sobre a distribuição de preservativos masculinos no ambiente escolar. Revista de Saúde Coletiva. 25 (2): 501-523.

Santos, M. P., et al. (2017). Pré-carnaval educativo sobre infecções sexualmente transmissíveis com adolescentes escolares. Rev enferm UFPE. 11(12), 511621.

Silva, D. M, Alves, M., Souza, T. O et al. (2013). Sexualidade na adolescência: relato de experiência. Rev enferm UFPE. 7(1):820-3.

Soares, C. B., Hoga, L. A. K., Peduzzi, M., Sangaleti C, Yonekura, T, \& Silva, D. R. A. (2014). Revisão integrativa: conceitos e métodos utilizados na enfermagem. Rev Esc Enferm USP. 48(2), 335-45.

Torres, R. A. M., et al. (2015). Comunicação em saúde: uso de uma web rádio com escolares. J. Health Inform. 7(2):58-61.

Xavier, L. D. A, Silva, C. F, Torres, E. F., et al (2017). Câncer de colo uterino e infecção sexualmente transmissível: percepção das mulheres privadas de liberdade. Rev enferm UFPE. 11(7), 2743-50. 